\title{
SOLAR SYSTEM OBJECTS OBSERVED BY HIPPARCOS.
}

\author{
D. HESTROFFER AND B. MORANDO \\ Bureau des Longitudes \\ 77 Av. Denfert Rochereau \\ F-75014 Paris
}

\begin{abstract}
The ESA satellite Hipparcos provides valuable photometric and astrometric results on minor planets and natural satellites. Observations of 48 asteroids, J II-Europa, S VI-Titan and S VIII-Iapetus were made from November 1989 to March 1993. A twenty seconds averaged normal place is constructed, providing thus positions accurate to a few hundredths of arcseconds and relative to a very precise and homogeneous reference frame.
\end{abstract}

\section{Introduction}

The systematic scanning of the sky by Hipparcos from November 1989 to March 1993 yields accurate astrometric parameters for about 120000 stars. It will provide an homogeneous optical counterpart of the ICRS's reference frame (ICRF). Because the Hipparcos sphere has no orientation, it is tied to the reference frame of extra-galactic objects. It can also be tied to the dynamical reference frame defined by the motion of solar system objects. Three natural satellites and 48 minor planets have been added to the list of stars to be observed by the Hipparcos satellite (they are listed on Table 1 and 2). These are small and bright objects not larger than $\approx 1$ ", and with magnitude lower than 12.5.

\section{Observations}

The payload design of the instrument and the global reduction of the FAST consortium are described in (Perryman et al., 1992) and (Kovalevsky et al., 1992); the reduction procedure of the astrometric data for minor planets is described in (Hestroffer et al., 1995). 
TABLE 1. Solar system objects observed by Hipparcos

Asteroids

\begin{tabular}{rlr|rlr|rlr}
\hline$N^{\circ}$ & Name & Nbr & N $^{\circ}$ & Name & Nbr & $N^{\circ}$ & Name & Nbr \\
\hline 1 & Ceres & 67 & 18 & Melpomene & 103 & 63 & Ausonia & 13 \\
2 & Juno & 62 & 19 & Fortuna & 31 & 88 & Thisbe & 36 \\
3 & Pallas & 63 & 20 & Massalia & 61 & 115 & Thyra & 32 \\
4 & Vesta & 51 & 22 & Kalliope & 64 & 129 & Antigone & 43 \\
5 & Astraea & 80 & 23 & Thalia & 66 & 192 & Nausikaa & 32 \\
6 & Hebe & 93 & 27 & Euterpe & 36 & 196 & Philomela & 16 \\
7 & Iris & 66 & 28 & Bellona & 36 & 216 & Kleopatra & 20 \\
8 & Flora & 57 & 29 & Amphitrite & 63 & 230 & Athamantis & 35 \\
9 & Metis & 47 & 30 & Urania & 48 & 324 & Bamberga & 66 \\
10 & Hygiea & 47 & 31 & Euphrosyne & 15 & 349 & Dembowska & 96 \\
11 & Parthenope & 67 & 37 & Fides & 33 & 354 & Eleonora & 97 \\
12 & Victoria & 24 & 39 & Laetitia & 103 & 451 & Patientia & 29 \\
13 & Egeria & 36 & 40 & Harmonia & 105 & 471 & Papagena & 108 \\
14 & Irene & 47 & 42 & Isys & 50 & 511 & Davida & 65 \\
15 & Eunomia & 87 & 44 & Nysa & 53 & 532 & Herculina & 38 \\
16 & Psyche & 46 & 51 & Nemausa & 15 & 704 & Interamnia & 72 \\
\hline
\end{tabular}

TABLE 2. Solar system objects observed by Hipparcos

Satellites

\begin{tabular}{rlr|rlr}
\hline \multicolumn{3}{c}{ Jupiter } & \multicolumn{3}{c}{ Saturn } \\
$\mathrm{N}^{\circ}$ & Name & Number & $\mathrm{N}^{\circ}$ & Name & Number \\
\hline J II & Europa & 88 & S VIII & Titan & 111 \\
& & & S VIII & Iapetus & 65 \\
\hline
\end{tabular}

Due to the particular scanning law of the Hipparcos satellite, the amount of observations varies between the different objects (columns 'Nbr' and 'Number' of Tables 1 and 2). Moreover the observation of a solar system body occurs in a $86^{\circ}$ wide zone around the quadratures, which can yield a photocentre offset of a few mas for the largest planetoids.

Because the fundamental measure is a photon count, Hipparcos not only provides positions of the observed objects, but also magnitudes with a precision of a few $0.01 \mathrm{mag}$ (Mignard et al., 1992), (Morando and Mignard, 1993). So, reduced light curves for a few objects and phase curves between $15^{\circ}$ and $25^{\circ}$ can be constructed. 


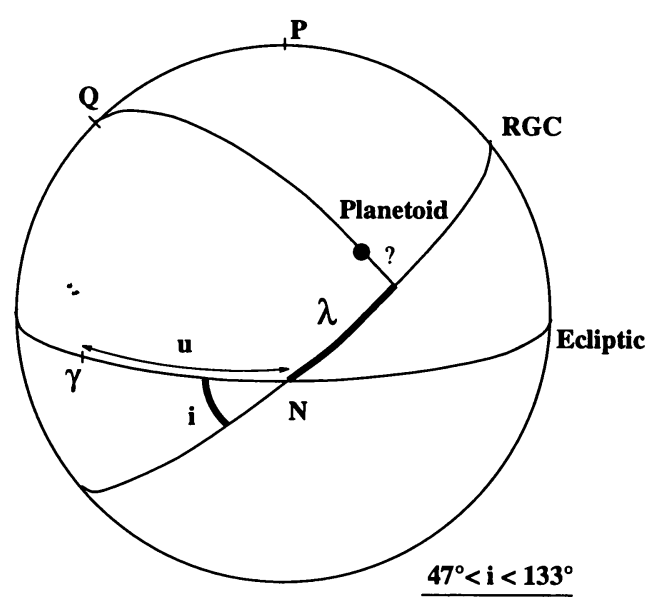

Figure 1. Position of a planetoid given by Hipparcos

\section{Astrometry}

As the measures are made through a modulating grid with parallel slits, Hipparcos observations only provide a one-dimensional abscissa $\lambda$ on a reference great circle (RGC) as shown on Figure 1. This precessing great circle oscillates, with a period of 57 days, around a plane perpendicular to the ecliptic, yielding thus more information on the planetoids latitude than on their ecliptic longitudes.

The reduction procedure takes into account effects of the order of 1 mas. Care has also been taken to avoid modelisation errors of such an amplitude so that no correction of the photocenter offset has been added. The given abscissa corresponds then to the astrometric direction of the planet photocentre for each transit in the field of view. In general, this normal point is distant to the center of mass by only a few 0.1 mas. The accuracy of the position obtained depends on the body's magnitude and size, it is of the order of 0":015.

The O-C's obtained, for the satellite S VI-Titan and for all the minor planets, from the comparison to the Hipparcos observations are shown on Figure 2. These are differences on the great circles; the dispersion present on these graphs arises from the measurement noise, the variation over successive transits on a same great circle, and, on a larger time scale, from the geometry of the scanning direction relative to the planets' orbit.

The calculated places for the minor planets were obtained by numerical integration with initial conditions taken from the "Ephemerides of minor planets, 1992". The O-C's depend on a rotation of the Hipparcos sphere 

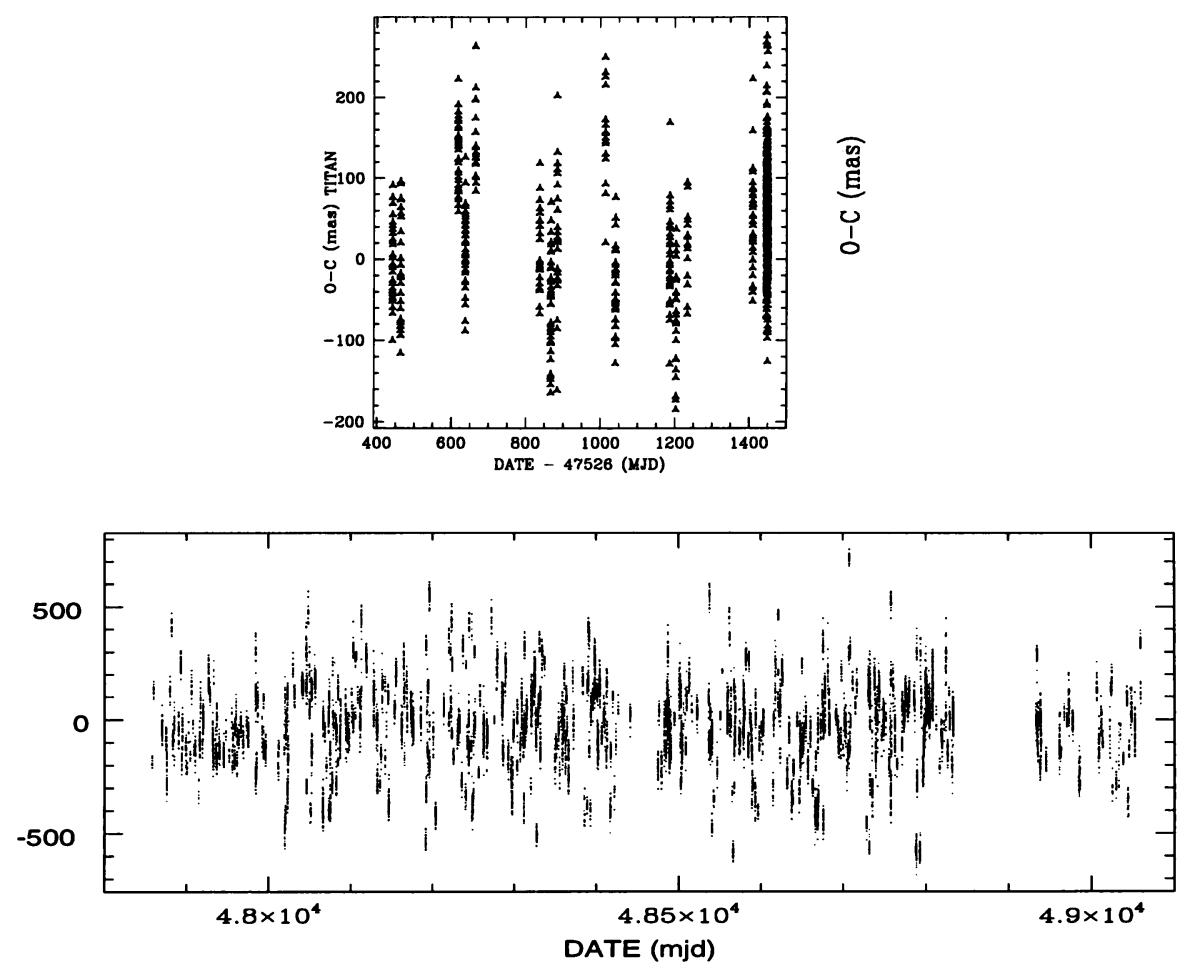

Figure 2. O-C's for S VI-Titan (top) and for the 48 minor planets (bottom).

relative to the dynamical reference frame, on the systematic errors due to phase effect, on errors of the Earth's ephemerides; but mostly on the initial conditions from the minor planets' ephemerides. For Titan the ephemerides were taken from the theory of Dourneau (1987) and the ephemerides of DE200 for Saturn, the O-C's are then in the range \pm 0 ".2; next step will be to compare these observations to the more recent theories TASS (Duriez and Vienne, 1991) or of Dourneau (1993), with improved ephemerides of Saturn.

\section{Reference Frame}

The link of the Hipparcos sphere to the dynamical frame - defined by the minor planets' motions - is given by an infinitesimal time dependant rotation: $\mathbf{W}=\mathbf{W}_{o}+\left(t-t_{o}\right) \cdot \mathbf{W}_{1}$. Other parameters outlined in Section 3 are the initial conditions of the minor planet ephemerides. With 3 years of ob- 
servations it is not possible to determine the whole set of initial conditions. Parameters such as eccentricity, semi-major axis or mean longitude are not or are poorly determined; nevertheless, the orientation of the osculating plane can be provided to an accuracy of a few mas.

Correction to the orientation of the Earth's osculating plane can not be determined simultaneously with those of the minor planets and the orientation of the sphere (Hestroffer et al., 1995). The orientation $\mathbf{W}_{o}$ and the rotation rate $W_{1}$, given with respect to the $\mathrm{DE} 200$ system, are provided with an accuracy of 1-2 mas and 1-3 mas/year respectively; where the orientation of the ecliptic is better determined than the position of the equinox (Hestroffer, 1994).

\section{Conclusion}

Hipparcos observations of minor planets and natural satellites, made over a period of 3 years, yield magnitude determination with a precision of a few $0.01 \mathrm{mag}$ and astrometric positions accurate to about 0".015. From the minor planets' astrometric data, it is possible to obtain a partial improvement of their ephemerides. These observations can however profitably complete the ground-based observations made on a wider time span. It is also possible to determine the rotation of the Hipparcos sphere relative to a dynamical reference frame with a precision of 3 mas and 3 mas/year.

Hipparcos observations of solar system objects are of great value for orbital improvement or frames linking, but they are spread over a short time span when compared to the minor planets' sidereal periods. These observations will be advantageously completed by high precision observations from ground (CCD observations of occultations or close approaches) or by astrometry from space (HST, Struve, GAIA).

\section{References}

Dourneau G. (1987) “Observations et étude du mouvement des huit premiers satellites de Saturne", Thèse, Bordeaux.

Dourneau G. (1993) "Orbital elements of the eight major satellites of Saturn determined from a fit of their theories of motion to observations from 1886 to 1985 ", $A \& A, 167$, 292-299.

Duriez L. and Vienne A. (1991) "A general theory of motion for the eight major satellites of Saturn", $A \& A, 243,263-275$.

Ephemerides of minor planets for 1992, Institute of Theoretical Astronomy, Leningrad.

Hestroffer D. (1994) “Astrométrie et photométrie des astéroïdes observés par le satellite Hipparcos. Apport à l'élaboration d'un système de référence dynamique”, Thèse, Observatoire de Paris.

Hestroffer D., Morando B., Mignard F., Bec-Borsenberger A. (1995) "Astrometry of minor planets with Hipparcos", accepted by $A \& A$.

Kovalevsky J., Falin J.L., Pieplu J.L., Bernacca P.L., Donati F., Froeschlé M., Galligani I., Mignard F., Morando B., Perryman M.A.C, Schrijver H., van Daalen D.T., 
van der Marel H., Villenave M., Walter A.G., Badiali M., Borriello L., Brouw W.N., Canuto E., Guerry A., Hering R., Huc Cl., Iorio-Fili D., Lacroute P., Lattanzi M., Le Poole R.S., Murgolo F.P., Preston R.A., Röser S., Sansò F., Wielen R., Belforte P., Bernstein H.H., Bucciarelli B., Cardini D., Emanuele A., Fassino B., Lenhart H., Lestrade J.F., Prezioso G. and Tommasini Montanari T. (1992) "The FAST Hipparcos Data Reduction Consortium: overview of the reduction software", $A \& A$.,258, 7-17.

Mignard F., Froeschlé M. and Falin J.L. (1992) "Hipparcos photometry: FAST main mission reduction", $A \& A ., 258,142-148$.

Morando B. and Mignard F. (1993) In:Developments in Astrometry and their impacts on Astrophysics and Geodynamics, I.I. Mueller and B. Kolaczek (eds.), p 2.

Perryman M.A.C, Høg E., Kovalevsky J., Lindegren L., Turon C., Bernacca P.L., Crézé M., Donati F., Grenon M., Grewing M., van Leeuwen F., van der Marel H., Murray C.A., Le Poole R.S. and Schrijver H. (1992) "In-orbit performance of the Hipparcos astrometry satellite", $A \& A ., 258,1-6$. 